\title{
Dipole Scattering and Its Implications for Vibrational Mapping at Lattice Resolution
}

\author{
Christian Dwyer ${ }^{1}$ \\ 1. Department of Physics, Arizona State University, Tempe, Arizona 85287, USA.
}

The recent capability of vibrational spectroscopy in the transmission electron microscope continues to attract significant interest [1]. Notwithstanding the obvious importance of the energy resolution, of intrinsic relevance to many electron microscopists is the spatial resolution with which the technique can be performed. This question is especially astute in light of the large degree of inelastic delocalization that can be expected from such low energy excitations $(\sim 100 \mathrm{meV})$. Here we consider this question in the context of vibrational spectroscopic imaging of a crystalline material, whereby the STEM-EELS geometry is used to generate a "vibrational map" of the sample.

The issue of inelastic delocalization is tied up with the notion of dipole scattering. In vibrational spectroscopy, dipole scattering arises from the long-wavelength optical-type vibrations in a polar material or molecule. In the electrostatic approximation such vibrations produce long-ranged "dipole" fields. In a crystalline sample, or for a large molecule, these fields build up and can cause the incident electrons to scatter inelastically at large impact parameters.

Here, using theory, we assess the effect of dipole scattering on vibrational mapping of a crystalline sample at lattice resolution [2]. The lattice-resolution vibrational signal arises predominantly from "impact" scattering from shorter-wavelength acoustic- and optical-type modes. The affect of the dipole scattering then is to produce a slowly-varying background in the lattice resolution maps. The relative strength of the dipole background is crucial, since it may mask the higher resolution signals.

As case studies, we consider calculated spatially-resolved vibrational maps of graphene and single-layer hexagonal boron nitride (Figure 1). We use DFT and the finite-displacement method to calculate the vibrational modes, and inelastic multislice theory to compute the electron scattering. We assume an aberration-free $60 \mathrm{keV}$ beam with a $30 \mathrm{mrad}$ convergence semi-angle. The maps assume an energy window of 150-200 meV (1200-1600 $\left.\mathrm{cm}^{-1}\right)$, which includes contributions mainly from the highestenergy optical phonons in each material.

Since graphene is non-polar, its optical modes do not give rise to significant long-ranged fields. Hence the scattering from these modes is similar to that from acoustic-type modes, which is highly-localized about the atoms and has a correspondingly smaller scattering cross section. In BN, an important consequence of a strong dipole background is that more incident electrons $N_{\mathrm{e}}$ will be needed to observe the lattice contrast in an experiment. For graphene, $N_{\mathrm{e}}$ is in the range $10^{4}-10^{5}$ (large doses reflecting the weak vibrational scattering from a single atomic layer). For BN, a polar material, the dipole background means that $N_{\mathrm{e}}$ is about 10 times larger again. For polar materials, a significant reduction of the dose can be achieved using an annular collection geometry [2].

[1] O.L. Krivanek, T.C. Lovejoy, N. Dellby, T. Aoki, R.W. Carpenter, P. Rez, E. Soignard, J. Zhu, P.E. Batson, M. Lagos, R.F. Egerton, and P.A. Crozier, Nature 514 (2014), 209; T. Miyata, M. Fukuyama, A. Hibara, E. Okunishi, M. Mukai, and T. Mizoguchi, Microscopy 63 (2014), 377; P.A. Crozier, K. March, 
and Q. Liu, Ultramicroscopy 169 (2016), 30; C. Dwyer, T. Aoki, P. Rez, S.L.Y. Chang, T. C. Lovejoy, and O. L. Krivanek, Phys. Rev. Lett. 117 (2016), 256101; P.A. Crozier, Ultramicroscopy 180 (2017), 104; P. Rez, T. Aoki, K. March, D. Gur, O.L. Krivanek, N. Dellby, T.C. Lovejoy, S.G. Wolf, and H. Cohen, Nature Comm. 7 (2016), 10945; G. Radtke, D. Taverna, M. Lazzeri, and E. Balan, Phys. Rev. Lett. 119 (2017)., 027402; M. J. Lagos, A. Trugler, U. Hohenester, and P.E. Batson, Nature 543 (2017), 529; A.A. Govyadinov, A. Konecna, A. Chuvilin, S. Velez, I. Dolado, A.Y. Nikitin, S. Lopatin, F. Casanova, L. E. Hueso, J. Aizpurua, and R. Hillenbrand, Nature Comm. 8 (2017), 95.

[2] C. Dwyer, Phys. Rev. B 96 (2017), 224102.

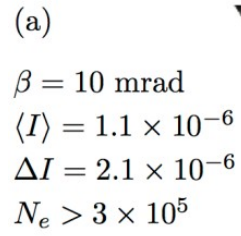

(b)

$$
\begin{aligned}
& \beta=30 \mathrm{mrad} \\
& \langle I\rangle=6.7 \times 10^{-6} \\
& \Delta I=8.6 \times 10^{-6} \\
& N_{e}>9 \times 10^{4}
\end{aligned}
$$

(c)

$\beta=50 \mathrm{mrad}$

$\langle I\rangle=1.7 \times 10^{-5}$

$\Delta I=3.3 \times 10^{-5}$

$N_{e}>2 \times 10^{4}$

(d)

$\beta=90 \mathrm{mrad}$
$\langle I\rangle=3.3 \times 10^{-5}$
$\Delta I=7.6 \times 10^{-5}$
$N_{e}>6 \times 10^{3}$

(e)

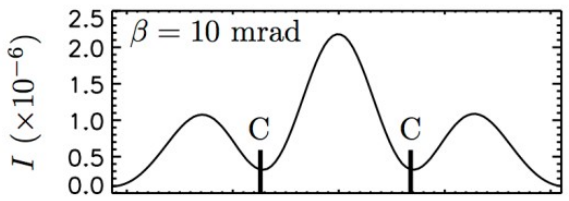

(f)

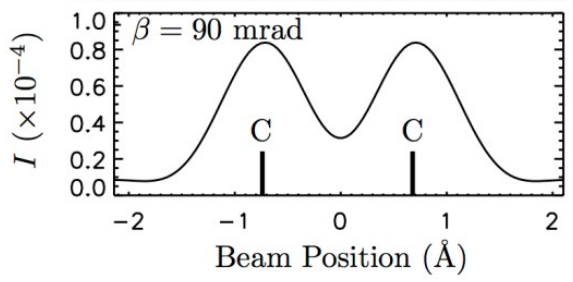

(g)

$\beta=10 \mathrm{mrad}$

$\langle I\rangle=1.2 \times 10^{-5}$

$\Delta I=2.0 \times 10^{-6}$

$N_{e}>3 \times 10^{6}$

(h)

$\beta=30 \mathrm{mrad}$

$\langle I\rangle=1.1 \times 10^{-4}$

$\Delta I=9.3 \times 10^{-6}$

$N_{e}>1 \times 10^{6}$

(i)

$\beta=50 \mathrm{mrad}$

$\langle I\rangle=1.2 \times 10^{-4}$

$\Delta I=3.3 \times 10^{-5}$

$N_{e}>1 \times 10^{5}$

(j)

$\beta=90 \mathrm{mrad}$

$\langle I\rangle=1.3 \times 10^{-4}$

$\Delta I=7.5 \times 10^{-5}$

$N_{e}>2 \times 10^{4}$
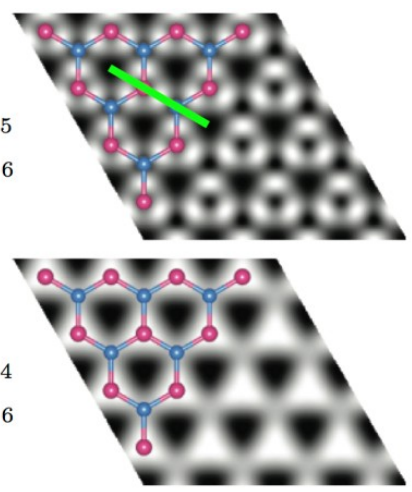

(k)

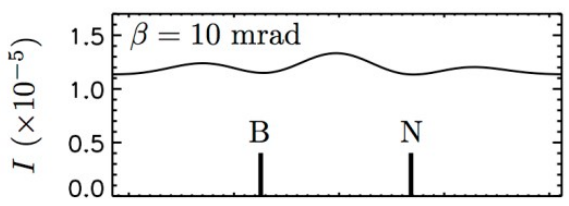

(l)
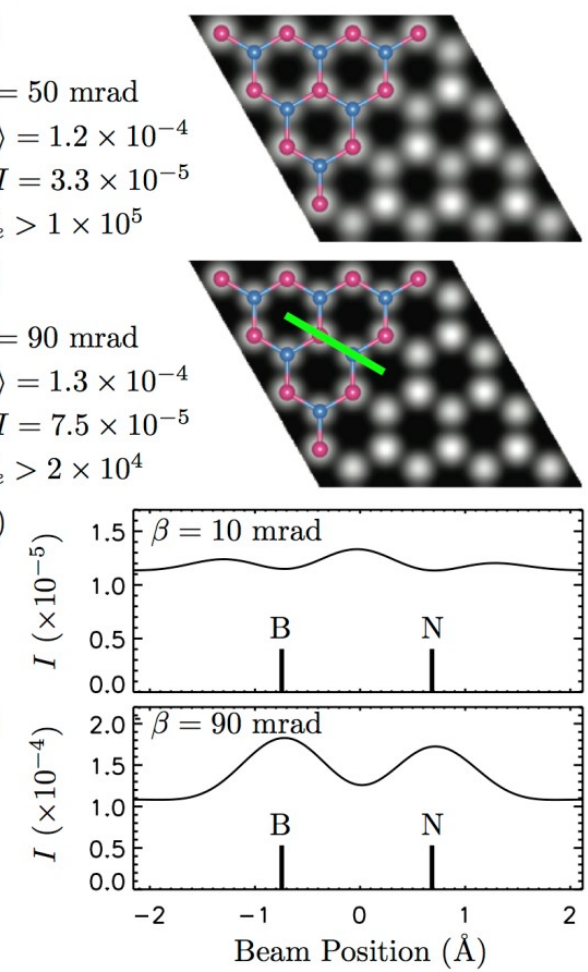

Figure 1. Calculated vibrational-spectroscopic images and line traces of graphene (a)-(f) and singlelayer hexagonal BN (g)-(1) with atomic models overlaid (for BN, B is red, $\mathrm{N}$ is blue). The beam paths for line traces are indicated by the green lines. Indicated for each image are the collection semi-angle $\beta$, the mean intensity $\langle I\rangle$ and magnitude $\Delta I$ of atomic scale contrast in units of probability per incident electron, and the number of incident electrons per pixel $N_{\mathrm{e}}$ required to detect the lattice contrast in the presence of Poisson noise. The images of BN contain a strong background intensity due to dipolar scattering, as evident in (k) and (1). 